\title{
ON and OFF Pathways Segregated at the Auditory Thalamus of the Guinea Pig
}

\author{
Jufang He \\ Department of Rehabilitation Sciences, The Hong Kong Polytechnic University, Hung Hom, Kowloon, Hong Kong, China
}

ON and OFF auditory responses were examined on the medial geniculate body (MGB) of the guinea pig. Single-unit and multiunit recordings were performed on 13 anesthetized subjects while either noise-burst or pure-tone stimuli were applied to the ear contralateral to the recorded hemisphere. Of 2187 unevenspaced samples, 1142 were ON neurons, 386 were OFF neurons, and 488 were ON-OFF neurons. Neither ON nor OFF neurons changed their response patterns when the stimulus was changed from a noise burst of $60 \mathrm{~dB}$ sound pressure level intensity (expressed in decibels per $20 \mathrm{mPa}$ ) to pure tones or noise bursts of other intensities. However, most of the ON-OFF neurons changed to either ON or OFF responses with some stimuli. OFF neurons formed clusters. With anatomical confir- mation, we found that OFF neuron clusters are always segregated from $\mathrm{ON}$ neuron clusters and form OFF sheets in various divisions of the MGB. In the ventral division of the MGB (MGv), the neurons showed mainly ON responses in its core and OFF responses in its periphery or on its boundary with other divisions. The MGv was partially surrounded by an OFF sheet dorsolaterally at the caudal part and medioventrally at the rostral part and was almost completely surrounded by an OFF sheet at the central level rostrocaudally.

Key words: medial geniculate body; OFF neurons; ventral division of the MGB; ON-OFF segregation; extracellular recording; auditory pathway
OFF neurons were first demonstrated in the visual system in the 1930s and were intensively studied thereafter (Hubel and Wiesel, 1961; Singer and Creutzfeldt, 1970; Schiller, 1982; Duysens et al., 1996; Bisti et al., 1998). Visual ON and OFF layers were clearly demonstrated in the lateral geniculate nucleus (Schiller and Malpeli, 1978; Le Vay and McConnell, 1982; Stryker and Zahs, 1983; Jones, 1985).

OFF neurons in the auditory system were first reported in the bat and then in the cat auditory cortices and auditory pathways (Suga, 1964; Kiang, 1965; Neuweiler et al., 1971; Grinnell, 1973; Aitkin and Prain, 1974; Suga et al., 1974, 1975; He et al., 1997). Although Suga and colleagues (Suga et al., 1974, 1975; Suga and Manabe, 1982) subsequently mentioned that the OFF neurons in the bat show their best frequency $(\mathrm{BF})$ between 58 and $62 \mathrm{kHz}$, hinting that OFF neurons might be site-specific, no report has clearly indicated that OFF neurons are spatially segregated from ON neurons in any nucleus of the auditory pathway or the auditory cortex.

In a recent study, we found that many OFF neurons in the cat dorsal auditory cortex, which is connected to the dorsal division of the medial geniculate body (MGB) and posterior nucleus (Po) of the thalamus, are stimulus duration selective (He et al., 1997; He and Hashikawa, 1998). Other studies have revealed that the OFF response is associated with sound source movement (Toronchuk et al., 1992; Spitzer and Semple, 1995).

Keeping the above knowledge in mind, we found that OFF

Received May 11, 2001; revised Aug. 21, 2001; accepted Aug. 21, 2001.

This study was supported by Hong Kong Research Grants Council Grant CERG PolyU5211/99M and by University Grant ASD EmW. I thank Edward G. Jones and Nobuo Suga for critical review of this manuscript and Christal Kam for technical assistance.

Correspondence should be addressed to Jufang He, Department of Rehabilitation Sciences, The Hong Kong Polytechnic University, Hung Hom, Kowloon, Hong Kong, China. E-mail: rsjufang@polyu.edu.hk.

Copyright (ㄷ) 2001 Society for Neuroscience $\quad 0270-6474 / 01 / 218672-08 \$ 15.00 / 0$ neurons always formed a cluster when we advanced the electrode throughout the MGB in a preliminary study. This preliminary result led to the present study to completely map the auditory thalamus in terms of $\mathrm{ON}$ and OFF neurons.

\section{MATERIALS AND METHODS}

Animal preparation. A total of 13 guinea pigs of both sexes, weighing 400-627 gm with clean external ears, served as subjects; normal auditory thresholds were estimated from the cortical unit responses. Anesthesia was initially induced with pentobarbital sodium (Nembutal; Abbott Labs, Irving, TX) (35 mg/kg, i.p.) and maintained by supplemental doses of the same anesthetic $\left(\sim 5-10 \mathrm{mg} \cdot \mathrm{kg}^{-1} \cdot \mathrm{hr}^{-1}\right)$ during the surgical preparation and recording. Atropine sulfate $(0.05 \mathrm{mg} / \mathrm{kg}$, s.c.) was given $15 \mathrm{~min}$ before anesthesia and at regular intervals $\left(0.01 \mathrm{mg} \cdot \mathrm{kg}^{-1} \cdot \mathrm{hr}^{-1}\right.$, s.c.) during the recording to inhibit tracheal secretion. The preparation of the guinea pig is similar to that of the cat, as has been described previously (He, 1997). Briefly, the subject was mounted in a stereotaxic device after the induction of anesthesia. A midline incision was made in the scalp, and a craniotomy was performed to enable us to vertically access the MGB in the left hemisphere. The dura mater was removed at a position vertically above the auditory thalamus. The head was fixed with two stainless steel bolts to an extended arm from the stereotaxic frame using acrylic resin, before the right ear was freed from the ear bar, so that the subject's head remained fixed to the stereotaxic device without movement. The procedures were approved by the Animal Subjects Ethics Subcommittee of The Hong Kong Polytechnic University.

Acoustic stimulus. Acoustic stimuli were generated digitally by a MALab system (Kaiser Instruments, Irvine, CA), which was controlled by a Macintosh computer (Semple and Kitzes, 1993; He, 1997). Acoustic stimuli were delivered to the subject via a dynamic earphone (DT-48; Bayer, Wuppertal, Germany) mounted in a probe. The sound pressure level (SPL) of the earphone was calibrated over a frequency range of 100 to $35 \mathrm{kHz}$ under computer control using a condenser microphone (Brüel and Kjaer one-fourth inch; Brüel and Kjaer Instruments, Marlborough, MA). The subject was placed in a double-walled soundproof room (NAP, Clayton, Australia). Repeated noise bursts and pure tones of varied durations with $\geq 1 \mathrm{sec}$ intervals and a $5 \mathrm{msec}$ rise-fall time were used to examine the ON and OFF neurons. Repeated pure tones of $\geq 400 \mathrm{msec}$ intervals were used to characterize the BFs of the recorded neurons.

Recording. Platinum or tungsten microelectrodes with impedances of 
9-12 M $\Omega$ (Frederick Haer and Co., Brunswick, ME) were advanced by a stepping-motor microdrive, which was controlled outside the soundproof room. The time of spike occurrence relative to stimulus delivery was stored in the same computer used as the stimulus controller by the MALab software. The computer automatically created raster displays and peristimulus time histograms (PSTHs) of the responses, together with frequency response functions (responses to pure tones plotted as a function of frequency).

The MGB was accessed vertically from the top of the brain in the stereotactically positioned subject. The penetrations were made according to a guinea pig brain atlas (Rapisarda and Bacchelli, 1977). The vertical coordinate of the electrode was determined at a point slightly above the cortical surface at the first penetration. A single electrode was used for each experiment, so that the depth coordinates could be kept consistent for different penetrations during the experiment. This technique enabled us to reconstruct a physiological map of a whole sagittal or frontal MGB plane containing many penetrations, by applying only a single lesion at the last penetration.

We isolated single units in the MGB during our recording by an amplitude and time window-discriminator. Multiunit recordings of three or less units were used in some cases and were not distinguished from single-unit recordings.

The duration of the stimulus was changed between 10 and $700 \mathrm{msec}$ at least once for every OFF or ON-OFF neuron to distinguish the OFF response from the long-latency or rebound responses.

Data processing. Recordings were sampled unevenly, emphasizing OFF responses. However, we considered this factor when we were calculating the spatial distribution of the ON and OFF neurons, by adding a weighting index on the sampling steps. ON-OFF neurons were calculated as partial $\mathrm{ON}$ and partial OFF neurons based on their proportions of $\mathrm{ON}$ and OFF firing rates. We defined the proportion of the sum of OFF neurons over the total number of the ON and OFF neurons as the OFF response ratio. The OFF response ratio was calculated for subjects for which the total recording distance of auditory responsive neurons over repeated penetrations was $>9.0 \mathrm{~mm}$.

Anatomical confirmation. After the recording session, the subjects were deeply anesthetized with sodium pentobarbital and perfused transcardially with $0.9 \%$ saline followed by a mixture of $0.4 \%$ paraformaldehyde and $2.5 \%$ glutaraldehyde in $0.1 \mathrm{~m}$ phosphate buffer, $\mathrm{pH}$ 7.3. The brains were removed and stored overnight in $0.1 \mathrm{M}$ phosphate buffer containing $30 \%$ sucrose. The thalami were cut transversally into $40-\mu \mathrm{m}$-thick sections using a freezing microtome. All sections were stained using the Nissl method. The Nissl sections were superimposed with the physiology map, using the electrode penetration tracks and the lesion for guidance. There was some shrinkage of the sections after the Nissl procedure. Enlargements of $10-13 \%$ of the Nissl images were made to match them to the physiology maps. Seven subjects were used for the purpose of anatomical confirmation.

\section{RESULTS}

The data presented here are from 2187 MGB neurons recorded from 13 anesthetized guinea pigs. Of 2187 uneven-spaced samples, 1142 were ON neurons, 386 were OFF neurons, 488 showed both $\mathrm{ON}$ and OFF responses and thus were called ON-OFF neurons, and the remaining 182 were classified as others. Both $\mathrm{ON}$ and OFF neurons were found in all experiments.

Spatial distribution of the ON and OFF neurons was calculated for seven subjects. The mean of the OFF response ratios was $20.1 \%$, varying from 8.8 to $29.4 \%$. It varied greatly depending on the location of the sagittal or frontal plane sampled.

We adopted an arbitrary definition of $50 \mathrm{msec}$ after the onset of the stimulus as the latency criterion to define the ON neurons and $100 \mathrm{msec}$ after the offset of the stimulus for the OFF neurons. ON neurons whose shortest response to the stimulus was located outside the $50 \mathrm{msec}$ cutoff were classified as others.

Figure 1 shows the responses of one example each for $\mathrm{ON}$, $\mathrm{OFF}$, and $\mathrm{ON}-\mathrm{OFF}$ neurons. The $\mathrm{ON}$ neuron in Figure $1 \mathrm{~A}$ did not change its firing pattern to an OFF or ON-OFF response as we changed the stimulus and its intensity. It was tuned to $20 \mathrm{kHz}$, as shown in its tuning curve.

The OFF neuron in Figure $1 B$ responded only in the OFF phase of the stimuli with a short latency of $<20 \mathrm{msec}$ after the offset of the stimuli. It did not change to an $\mathrm{ON}$ or $\mathrm{ON}-\mathrm{OFF}$ response when we changed the frequency and intensity of the stimulus, as shown in the figure. The neuron showed a responsive threshold of $50 \mathrm{~dB}$ SPL and tuned to $6 \mathrm{kHz}$.

The ON-OFF neuron in Figure $1 C$, which exhibited an $\mathrm{ON}-$ OFF response to a noise burst of $60 \mathrm{~dB}$ SPL (data not shown), showed an $\mathrm{ON}-\mathrm{OFF}$ response to a $0.8 \mathrm{kHz}$ pure tone of $80 \mathrm{~dB}$ SPL (Fig. $1 C, 2$ ) and changed its firing pattern to an ON response only when we lowered its stimulus intensity to $40 \mathrm{~dB}$ SPL (Fig. $1 C$, $4)$. The neuron changed to an $\mathrm{ON}$ response for a pure-tone stimulus of low frequency at $0.2 \mathrm{kHz}$ (Fig. $1 C, 1$ ) and to an OFF response for that of higher frequency at $2.0 \mathrm{kHz}$ (Fig. 1C, 3). The ON response of the neuron was tuned to a lower frequency at a lower threshold than the tuning of the OFF response.

The firing patterns of the ON, OFF, and ON-OFF neurons were first defined under the noise-burst stimulus of $60 \mathrm{~dB}$ SPL and were examined under different stimuli of noise bursts and pure tones at varied intensities. Of $45 \mathrm{ON}$ neurons examined, none changed to an OFF or an ON-OFF response. Of $10 \mathrm{OFF}$ neurons, only one changed to an $\mathrm{ON}-\mathrm{OFF}$ response when we changed to pure tones as the stimuli. However, 14 of $18 \mathrm{ON}-\mathrm{OFF}$ neurons examined changed their response patterns to either an ON or OFF response with some stimuli.

The duration of the stimulus was changed to examine the OFF neurons from the long-latency neurons and to examine the ONOFF neurons from the ON neurons with a rebound response, as shown in the examples in Figure $1 D$.

\section{ON and OFF neurons along a vertical penetration}

Figure 2 shows neuronal responses along a vertical penetration. Of the 28 single units recorded in this penetration, 13 were classified as $\mathrm{ON}$ neurons, 9 as $\mathrm{OFF}$ neurons, 3 as $\mathrm{ON}-\mathrm{OFF}$ neurons, and 3 as others.

In this penetration, we observed two clusters of OFF neurons: one from neurons 7-10 and the other from neurons 22-26, separated by $\mathrm{ON}$ neurons. On the boundary of the OFF cluster, there were ON-OFF neurons, 6, 11, and 20, which define an ON-OFF interface if we assume provisionally that the ON and OFF neurons form ON and OFF layers. The ON neurons in the dorsal part of the penetration (neurons 1-5) showed relatively longer latencies, which became shorter [neurons 12-19, in the ventral division of the MGB $(\mathrm{MGv})]$ as the electrode traveled through the first OFF region. The neuronal responses after the second OFF region became weaker and more irregular during the repeated noiseburst stimuli (neurons 27 and 28). The OFF neurons in this penetration showed latencies between 20 and $45 \mathrm{msec}$ and a phasic firing pattern.

Depending on the position of the vertical penetration, the MGB could be divided into multiple ON-OFF layers. In the example shown in Figure 2, two ON and two OFF layers were distinguished. Compared with the ON layers, the OFF layers were much thinner. ON-OFF neurons were often observed between the $\mathrm{ON}$ and $\mathrm{OFF}$ layers.

\section{Maps of ON and OFF neurons in the MGB}

Seven complete and three partial frontal planes at various rostrocaudal levels were mapped for the $\mathrm{ON}$ and $\mathrm{OFF}$ neurons, with penetrations spaced between 150 and $500 \mu \mathrm{m}$ from each other. Four frontal planes are shown in Figures 3, 4A, and 5. Four complete and two partial parasagittal planes were also mapped, and an example is shown in Figure $4 B$. Seven brains were cut in 
Figure 1. Responses and tuning curves of ON, OFF, and ON-OFF neurons. Four PSTHs of responses to pure-tone stimuli of varying frequencies and intensities are shown for each neuron in $A$ $(\mathrm{ON}), B$ (OFF), and $C$ (ON-OFF). Their corresponding frequencies and intensities are indicated in their tuning curves on the right. Solid curves indicate the tuning curves of the $\mathrm{ON}$ responses and dotted curves indicate the tuning curves of the OFF responses. The responses of an OFF $(a)$ and an ONOFF $(b)$ neuron to noise bursts of varied durations are shown in $D$. The duration of the stimulus is shown under each display. Each stimulus was repeated 20 times at an interval of $1 \mathrm{sec}$, for which only the first $200-500 \mathrm{msec}$ is shown in the figure. The conventions described here apply to Figures 2 and 3.
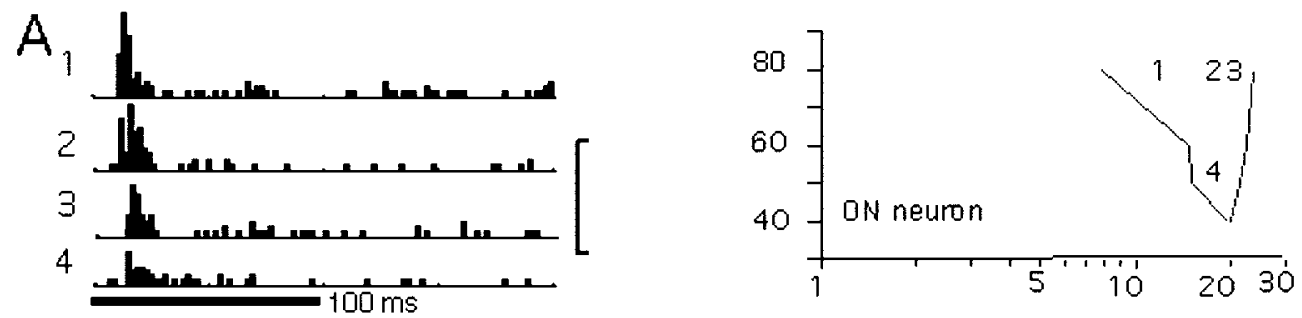

\section{$\mathrm{B}$}
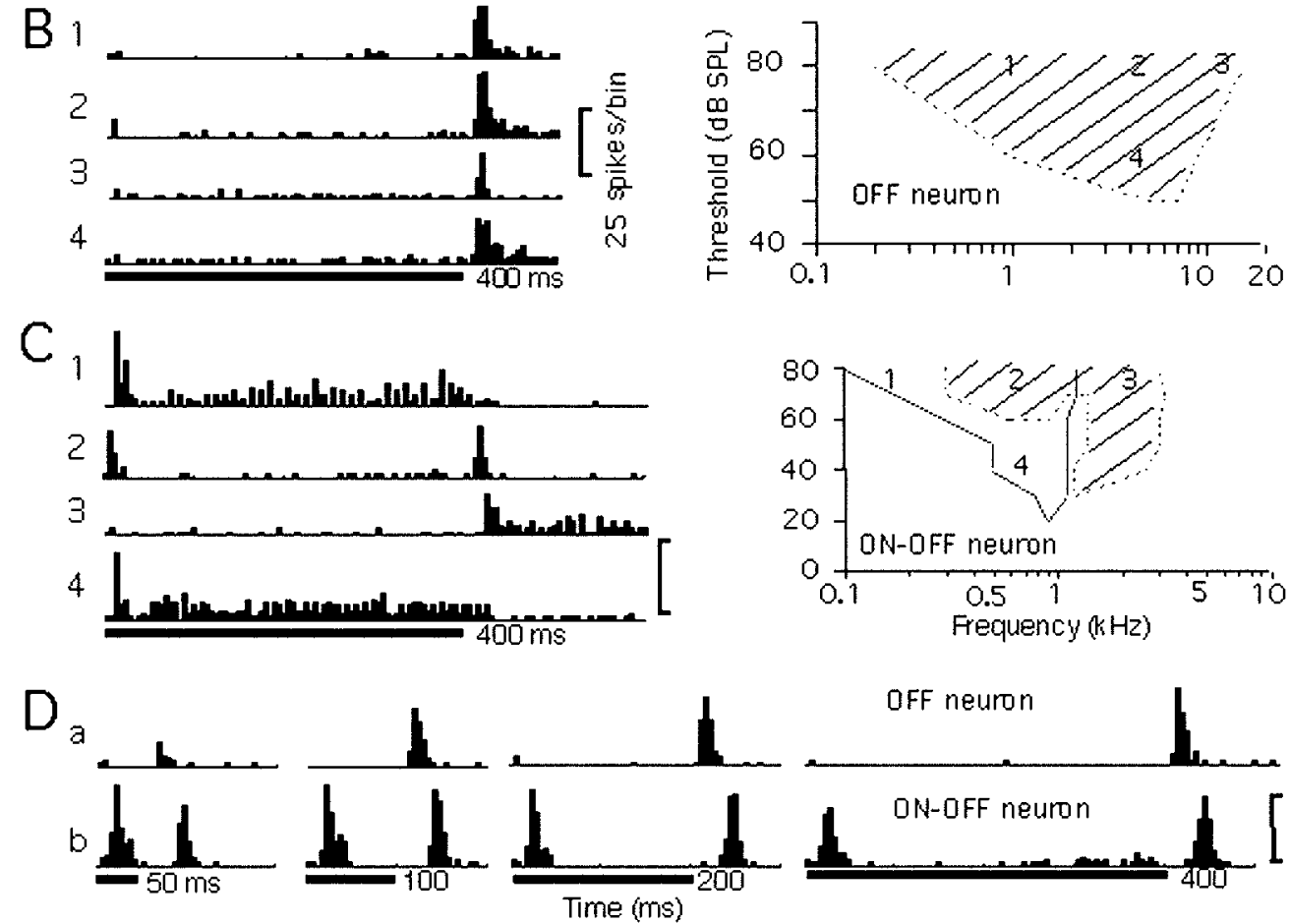

frontal sections and Nissl stained after physiological recording. The Nissl sections of the thalami were superimposed with the ON-OFF physiology maps of the frontal planes, two of which are shown in Figures 3 and 5. The electrode tracks in the Nissl sections agreed well with the coordinates of the physiological recording in all brains.

Figure 3 shows a frontal plane of $\mathrm{ON}$ and OFF neurons. The PSTHs shown in the figure were sampled from all over the OFF region. The sampled section in this figure was located $\sim 2.0 \mathrm{~mm}$ from the caudal pole of the MGB, and spanned $\sim 2.5 \mathrm{~mm}$ lateromedially and $2.0 \mathrm{~mm}$ dorsoventrally. A small lesion shown in the right bottom corner of the Nissl section and marked with an $X$ matched with the physiology penetrations very well. In general, the auditory responsive region is well matched with the Nisslstained MGB as defined by previous investigators (Rapisarda and Bacchelli, 1977; Redies et al., 1989; Redies and Brandner, 1991). An additional auditory responsive area was found located dorsomedially to the rostromedial division of the MGB (MGrm) and extended rostrally beyond the MGB. The area might be equivalent to the Po of the cat and thus is named Po in this report. Counting Po as a part of the auditory thalamus, it extended $\sim 3.3$ $\mathrm{mm}$ rostrocaudally for this subject.

Figure 4 shows two ON-OFF maps at frontal and sagittal planes which are perpendicular to each other. The frontal plane is located at $\sim 0.5 \mathrm{~mm}$ from the caudal pole of the MGB. Three clusters of OFF regions were found in this plane: one at dorsolateral, one at ventral, and one at dorsomedial. In the sagittal plane shown in Figure $4 B$, which could encompass part of all MGB divisions and the Po, only two OFF regions were found: one located caudally extending from the caudomedial division (MGcm) to the shell division (MGs) of the MGB and one ventrally in the MGrm region.

Figure 5 shows two frontal planes of ON-OFF maps combined with the Nissl sections. The auditory thalamus of this subject, examined by Nissl staining, extended $\sim 3.5 \mathrm{~mm}$ rostrocaudally. The section in Figure $5 A$ was located in the rostral MGB $\sim 0.8$ $\mathrm{mm}$ from the rostral pole of the auditory thalamus; the section in Figure $5 B$ was located in the caudal $\mathrm{MGB} \sim 1.1 \mathrm{~mm}$ from the caudal pole of the auditory thalamus and spanned $\sim 2.0 \mathrm{~mm}$ dorsoventrally.

Maps in Figures 3-5 showed clear segregations of the ON and OFF regions. OFF neurons formed one or more belts in every plane.

Another parasagittal plane was sampled at the level at which a frontal plane showed at least a clear OFF region in three experiments (data not shown). The belt-shaped OFF regions in the frontal plane extended rostrocaudally in the parasagittal plane, clearly indicating an OFF sheet rather than a belt or ring. This phenomenon was evident in two other experiments with both frontal and parasagittal planes, one of which is shown in Figure 4.

\section{Ventral division partially surrounded by OFF sheets}

The OFF neurons in Figure 3 formed two belts, of which one ran laterally; the other was bent in the ventromedial part of the MGB. 


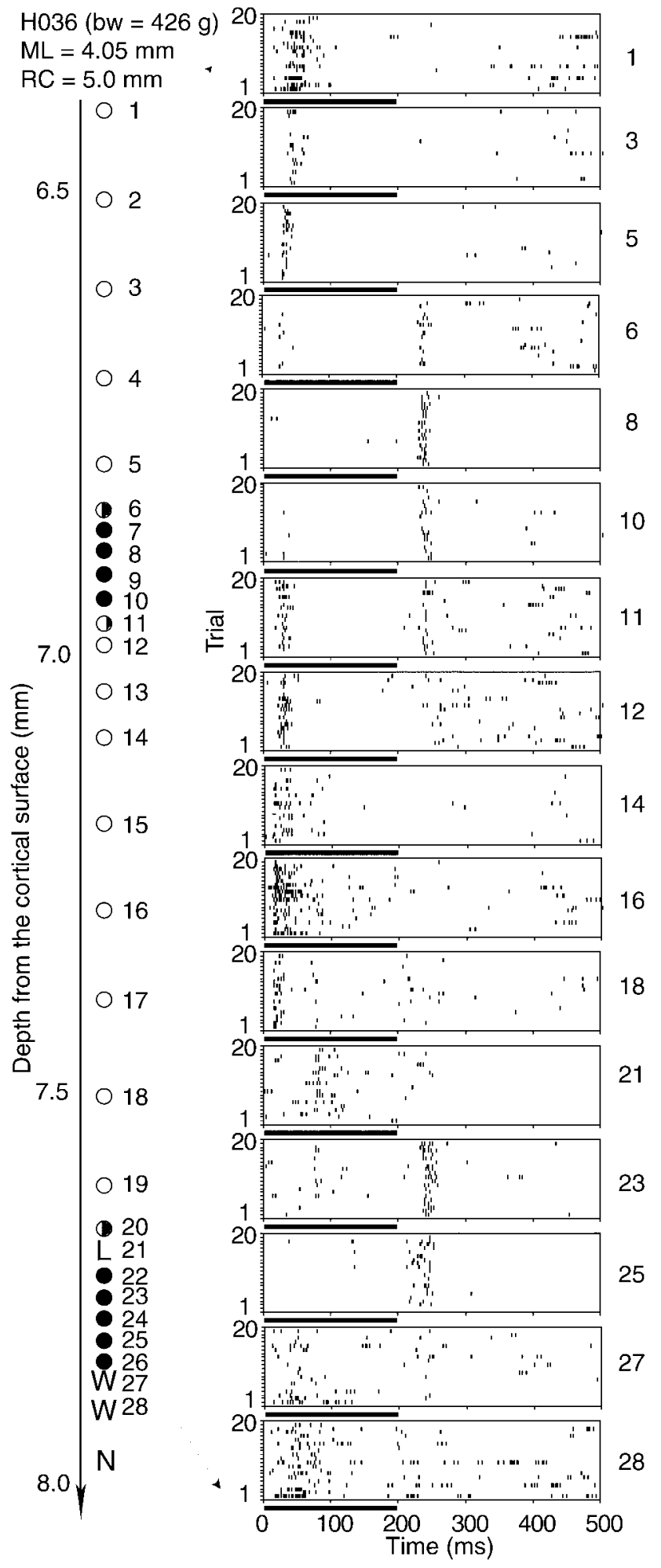

Figure 2. Raster displays of neuronal responses along a vertical penetration through the MGB. Noise bursts of $200 \mathrm{msec}$ duration and $60 \mathrm{~dB}$ SPL intensity were used as the stimuli for each neuron. The depth from the cortical surface of each neuron is shown to the left. The neurons were sampled at either 100 or $50 \mu \mathrm{m}$ intervals. The coordinates of the penetration and the body weight of the subject are shown at the top left. $b w$, Body weight; $M L$, mediolateral coordinate; $R C$, rostrocaudal coordinate. Filled circles represent OFF neurons, open circles represent ON neurons, and half-filled circles represent ON-OFF neurons. $L$, Long latency ( $>50$ $\mathrm{msec}) ; W$, weak response; $N$, no response. The conventions described here apply to Figures 3, 4, and 5.
The two belts might be weakly connected in the middle of the MGB, forming an $\mathrm{H}$-shaped OFF sheet, which divided the ON neurons in this plane into two large portions: one dorsomedially and one ventrolaterally.

By examining the Nissl section of the recorded frontal plane, in which the multiple electrode tracks were superimposed with the $\mathrm{ON}-\mathrm{OFF}$ map, the ventrolateral ON neurons were located in the MGv. The OFF belt lateral to the MGv, which formed a caudolateral sheet, was largely located in the MGs or partially in the MGv. The OFF responses in the dorsal part showed relatively longer latencies than those in the ventral part, as can be seen in the PSTHs in the figure.

The bending ventromedial OFF sheet was primarily located in the MGrm, on the borders between the MGv and MGrm and between the MGs and MGrm, and partially in the ventral part of the MGs.

The dorsolateral and the ventral OFF regions in Figure $4 A$ were located in the $\mathrm{MGv}$ and $\mathrm{MGcm}$ and covered the $\mathrm{MGv}$ dorsolaterally and ventrally. The OFF region at dorsomedial MGB was located at the MGcm and might be weakly connected with the ventral OFF region as shown in the parasagittal plane in Figure $4 B$.

In a more caudal plane as shown in Figure $5 B$, a relatively heavy sheet was found in the dorsolateral part of the MGB, covering a large region of the dorsal division (MGd) and the dorsolateral part of the relatively thick MGs and extending to the ventral part of the MGs. A few small OFF regions could be found in the ventral and dorsal parts of the MGB.

In another frontal plane near the rostral pole of the MGB (Fig. $5 A)$, an OFF belt was found starting from the MGs at the ventrolateral site, extending to the medial part ventrally and bending dorsally to the central part of the MGB. The belt surrounded approximately one-half of the $\mathrm{MGv}$ at this plane.

A series of sketches summarizes the OFF maps of all subjects, as shown in Figure $5 C$. The maps in Figure $5, A$ and $B$, correspond to sections 2 and 4 in Figure $5 C$. The map in Figure 3 is located approximately at the level of section 3 of Figure $5 C$. In summary, the dorsolateral OFF sheet in the MGcm and MGs, which was located in the caudal half of the MGB, was weakly connected to the medioventral OFF sheet, which was located in the rostral half of the MGB. The MGv was partially surrounded by an OFF sheet dorsolaterally at the caudal part and medioventrally at the rostral part, and was almost completely surrounded by an OFF sheet at the central level rostrocaudally.

\section{DISCUSSION}

\section{The auditory thalamus of the guinea pig}

In the present study, recording tracks and recording planes were located from lateral to medial and from rostral to caudal in the MGB. Best-frequency mapping and neuronal response latency information, together with the Nissl staining, permit the parcellation of the MGB, based on previous criteria set by Redies et al. (1989) and Redies and Brandner (1991). As a dorsal division, MGd was discriminated, as indicated in Figures 3-5. Another auditory responsive region, located dorsomedially to the MGrm near the rostral pole of the MGB and extending rostrally beyond the MGB, is probably equivalent to the Po of the cat. We confirmed the gradient of the tonotopic organization of the MGv: the BF increases mediolaterally and caudorostrally (Redies et al., 1989; Redies and Brandner, 1991). 


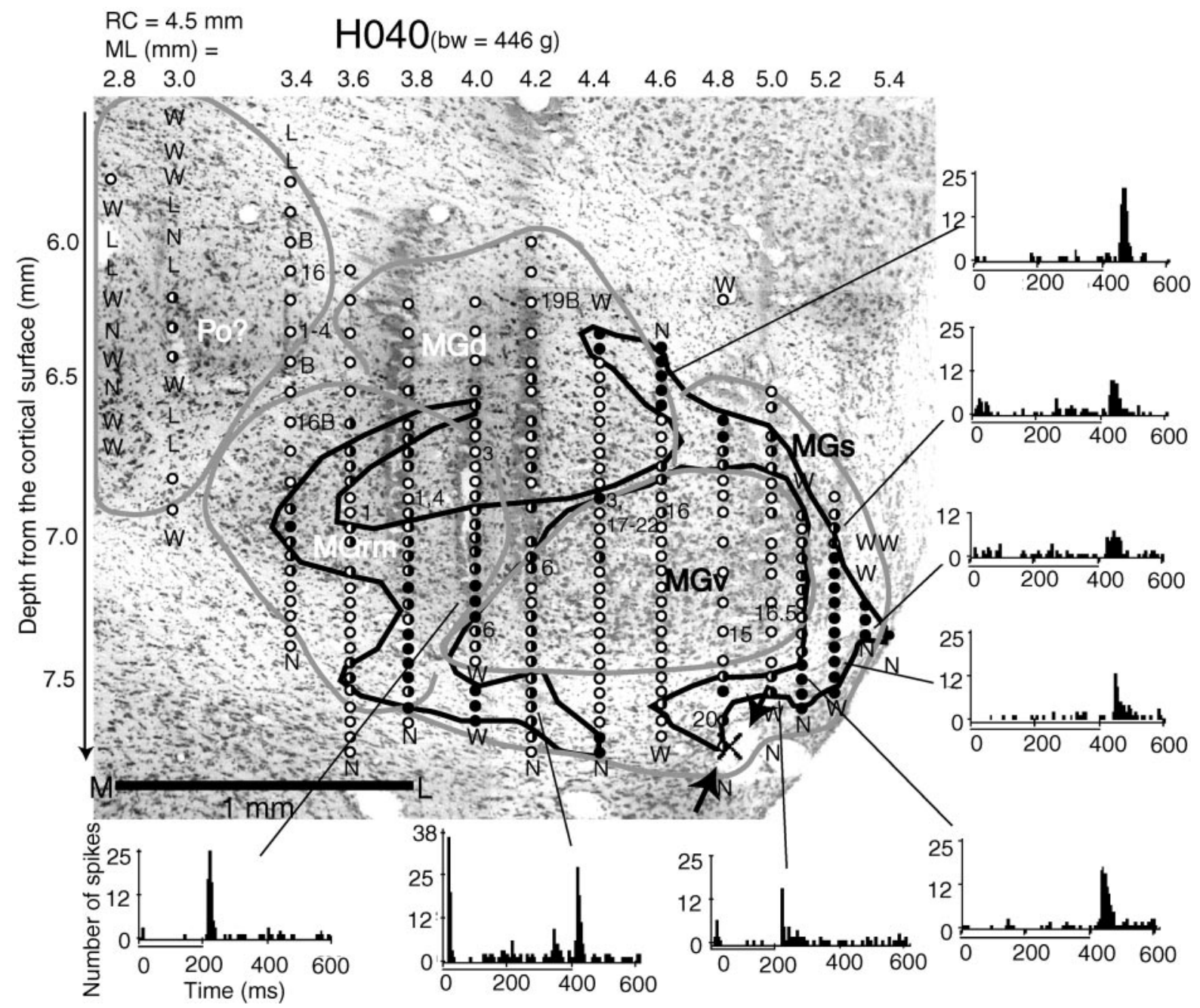

Figure 3. A frontal ON-OFF map superimposed with the Nissl section at the same level in subject H040. Using the coordinate of Rapisarda and Bacchelli (1977), the plane was at a rostrocaudal coordinate $(R C)$ of $4.5 \mathrm{~mm}$. The mediolateral coordinate is shown above the map and the depth coordinate is shown on the right. The depth coordinator was initiated at the first penetration and kept the same during the entire mapping process. Illustrative contours are used to circle the neighboring OFF neurons into bundles. Numbers indicate the best frequencies. $M$, Multipeaked tuning; $B$, broad tuning; $b w$, body weight. Arrows indicate the lesion made at the auditory responsive site of the last electrode penetration. The location for the recording penetration in the physiology map is marked with an $X$. Previous documents did not include the region marked $P o$ in the auditory thalamus of guinea pigs. However, the region was very likely equivalent to the Po of the thalamus in other species in terms of its location and responsiveness to sound stimuli. Superimposing the physiology map with the Nissl section, the displacement was smaller than $100 \mu \mathrm{m}$. The Nissl section was enlarged by $11.1 \%$ to match the marks of the electrode tracks with the stereotaxic coordinates of the physiological recording, because the brain sections shrank by that amount during Nissl processing. The peristimulus time histograms of eight OFF or ON-OFF neurons were sampled from varied locations on the OFF sheets. They were summations of responses to noise bursts of 20 trials and in $5 \mathrm{msec}$ bins. The conventions described here apply to Figures 4 and 5 .

\section{OFF responses}

In the visual system the proportion of OFF or ON-OFF neurons relative to ON neurons (Jassik-Gerschenfeld et al., 1976; Schiller and Malpeli, 1978, their Table 1) is much higher than that of OFF or ON-OFF neurons in the auditory system, in which only one-fifth of the responses are estimated to be OFF responses. This might explain why OFF neurons have received relatively less attention. Nevertheless, OFF neurons in the auditory system have been reported since as early as 1964 (Suga, 1964; Kiang, 1965; Neuweiler et al., 1971; Grinnell, 1973; Aitkin and Prain, 1974; Suga et al., 1974, 1975; He et al., 1997). The overall patterns of the OFF and ON-OFF responses in the bat's cochlear nerve fibers and auditory cortex (Suga and Manabe, 1982, their Figs. 2 and 3) were the same as those in the guinea pig's thalamus. However, the latency of the OFF response in the guinea pig's thalamus, usually $>15 \mathrm{msec}$, is much longer than that in the bat (for example, in the inferior colliculus, which is $\sim 10 \mathrm{msec}$ ) (Casseday et al., 1994, their Fig. 3).

Examined with different stimuli, our study clearly showed that the pure ON and OFF neurons defined under the stimulus of a 60 dB SPL noise burst did not change to other response patterns. Most ON-OFF neurons, however, might change to either $\mathrm{ON}$ or OFF responses as the stimulus changes. In the present study, the subjects were under pentobarbital anesthesia. According to Zurita et al. (1994), OFF neurons under pentobarbital anesthesia were less evident than under nitrous oxide anesthesia. Reviewing their examples, the OFF responses might have disappeared, but they did not change to ON responses (Zurita et al., 1994).

\section{ON and OFF segregation}

As mentioned above, ON and OFF or ON-OFF pathways in the visual system are segregated in the thalamus and primary visual 

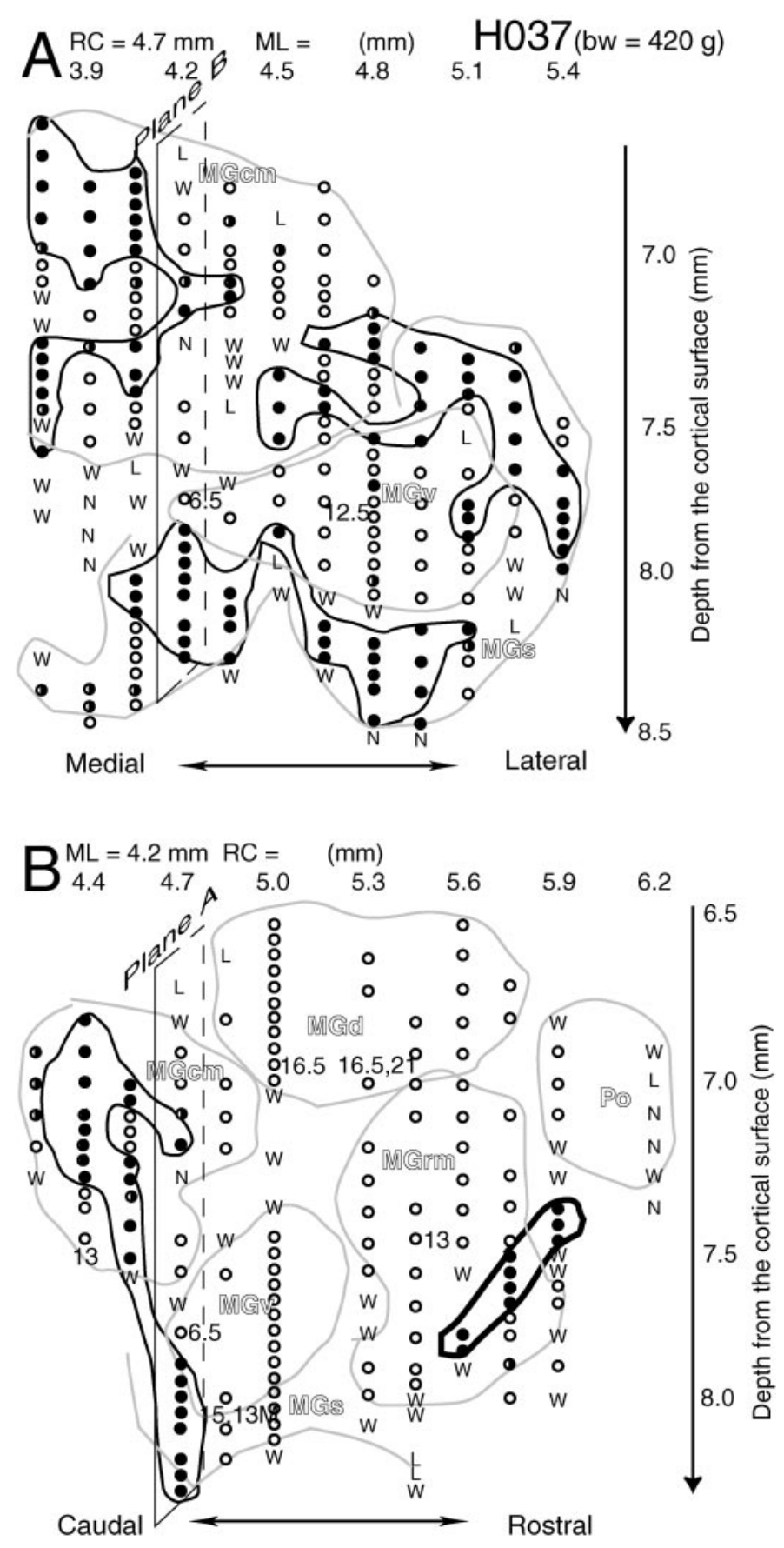

Figure 4. Mapping of ON and OFF neurons across frontal and sagittal planes of the MGB for subject H037. The frontal plane [rostrocaudal coordinate $(R C)$ of $4.7 \mathrm{~mm}$ ] is shown in $A$ and the sagittal plane [mediolateral coordinate $(M L)$ of $4.2 \mathrm{~mm}$ ] is shown in $B$. The intersections of the sagittal plane ( plane $B$ ) and the frontal plane (plane $A$ ) are marked with parallelograms in $A$ and in $B$, respectively. The mediolateral coordinates for the frontal plane $(A)$ and the rostrocaudal coordinate for the sagittal plane $(B)$ are shown above the maps; the depth coordinates for both maps are shown on the right. $b w$, Body weight.

cortex (Schiller and Malpeli, 1978; Stryker and Zahs, 1983; Bowling and Michael, 1984; Norton et al., 1985). In the lateral geniculate nucleus of carnivores, OFF layers and ON layers alternate, with the ventral parts of the $\mathrm{A}$ and $\mathrm{A}_{1}$ laminas being dominated by OFF responses and the dorsal parts dominated by $\mathrm{ON}$ responses (Schiller and Malpeli, 1978; Le Vay and McConnell,
1982). The OFF layers are always concentrated in the external half of the geniculate lamina and the posterior leaflet (Le Vay and McConnell, 1982; Stryker and Zahs, 1983). This trend has also been found in the caudal and lateral portions of the MGB, where a thick OFF layer can be identified in the MGs and $\mathrm{MGcm}$ in all subjects.

It is very clear that the MGv is partially surrounded by one or more OFF or ON-OFF sheets. A dorsolateral sheet in the caudal pole of the MGB is located in the MGcm and extends to the MGs rostrally, thus covering half of the MGv. Another OFF sheet surrounds the MGv medioventrally in the rostral half. The two sheets might be connected with each other in the rostrocaudal middle part of the MGB. The OFF sheets are much thinner than the $\mathrm{ON}$ regions.

In the report by Suga and Manabe (1982), ON, ON-OFF, and OFF neurons were clearly demonstrated, and ON-OFF neurons were commonly observed between 58 and $62 \mathrm{kHz}$, with the implication that they were site-specific (Suga et al., 1975). In a penetration to the auditory thalamus (Calford and Webster, 1981, their Fig. 10A), two separate OFF neurons were recorded in the dorsocaudal nucleus of the cat MGB. Aitkin and Prain (1974) reported $\mathrm{ON}-\mathrm{OFF}$ neurons in the cat MGB with a suggestion of $\mathrm{MGv}$ and the medial division of the MGB.

Although some of the ON-OFF neurons could have resulted from separate $\mathrm{ON}$ and $\mathrm{OFF}$ neurons, most were from isolated single units. It is very common to see $\mathrm{ON}-\mathrm{OFF}$ neurons between the ON and OFF layers, as shown in Figures 2-5. This result is comparable with the visual system, where ON-OFF responses were subsequently found between the ON and OFF layers (Le Vay and McConnell, 1982; Stryker and Zahs, 1983).

\section{Physiological implications of the OFF neurons and OFF sheets}

The neurons in Figures $1, B$ and $D$, and neurons 8, 10, and 25 in Figure 2 had very few spontaneous spikes but showed strong OFF responses that were locked at the OFF phase with certain latencies after the offset of the stimulus. The cortical neurons in Figure 9 of our previous report (He et al., 1997) showed the same trend. These results support the theory that the OFF neurons are not simply rebounds preceded by suppression of background activity, as mentioned in a previous report (Suga, 1964). Inhibition during the presence of a stimulus might be one of the causes of OFF responses at the higher auditory pathway and cortex (Suga, 1973). Another cause of OFF responses was proposed to be mechanical OFF transience occurring in the inner ear (Suga et al., 1975).

The tuning of the OFF auditory brainstem response has been reported to be complementary to the tuning of the $\mathrm{ON}$ response (Henry, 1985). The complementary tuning characteristic of the $\mathrm{ON}$ and OFF responses was also confirmed at unit level in the auditory cortex (Pelleg-Toiba and Wollberg, 1989; Heil et al., 1992). This result is evident in the ON-OFF tuning curve of Figure $1 C$ and has been confirmed from 10 samples of $>18$ neurons or neuron pairs in our experiments (our unpublished results).

It is evident that the $\mathrm{ON}$ and $\mathrm{OFF}$ responses are correlated with the direction of movement of a sound source (Toronchuk et al., 1992). Selectivity for sound moving away from the head correlated with the OFF responses, whereas the ON responses correlated with a preference for moving the sound source toward the head. OFF neurons are also involved in the detection of interaural phase disparity (Spitzer and Semple, 1995, their Fig. 7). 


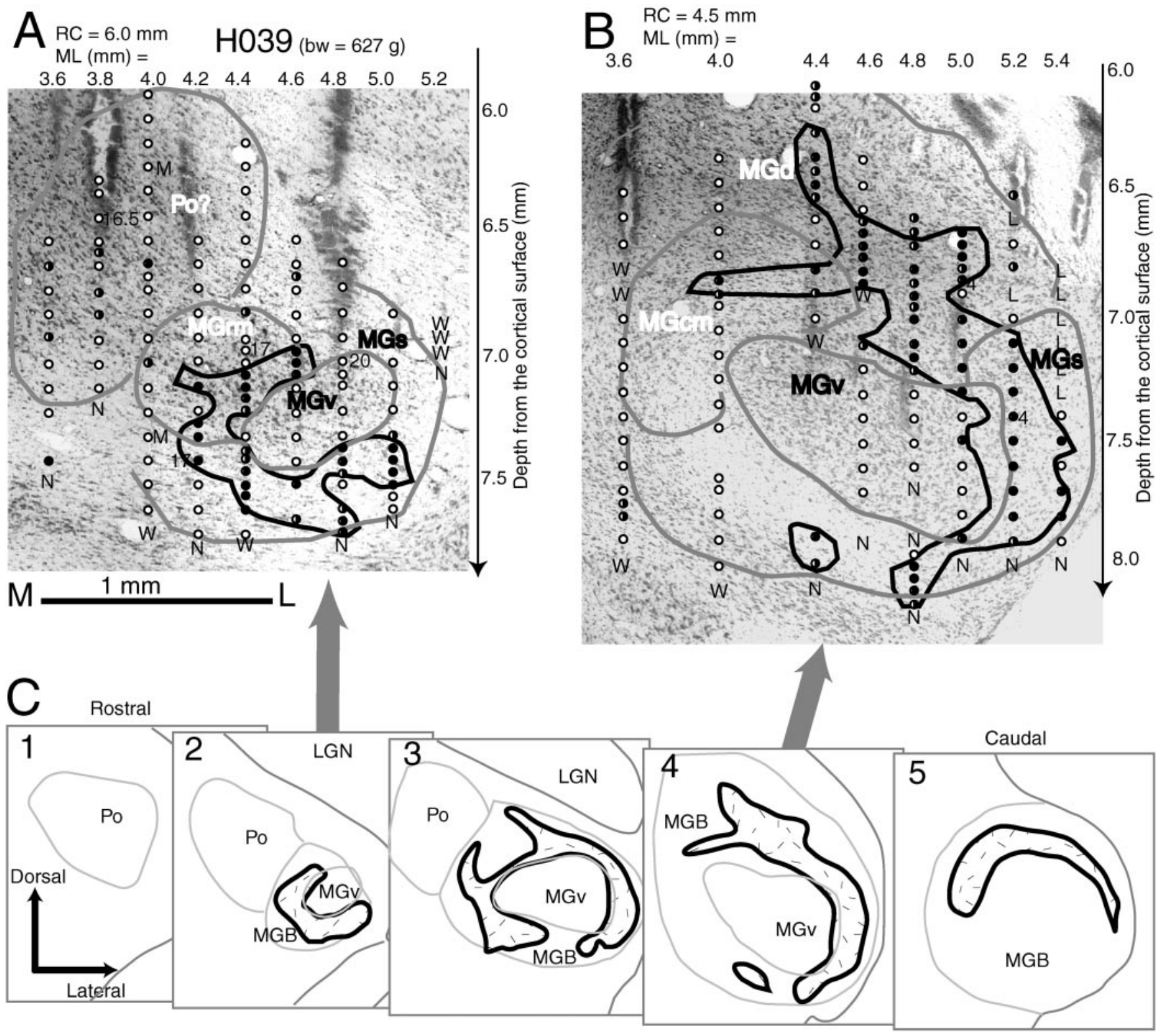

Figure 5. Two frontal ON-OFF maps of subject H039 superimposed with the Nissl sections at the same levels $(A, B)$ and a series of sketches of the OFF maps $(C)$. The rostral section $(A)$ was at a rostrocaudal coordinate $(R C)$ of $6.0 \mathrm{~mm}$; the caudal section $(B)$ was at a rostrocaudal coordinate of 4.5 $\mathrm{mm}$. The Nissl sections of this subject were enlarged by $11.9 \%$ to match the marks of the electrode tracks with the stereotaxic coordinates of the physiological recording. Most electrode tracks are identical in the Nissl section and match the stereotaxic coordinates. A series of sketches in the auditory thalamus were drawn from the Nissl sections of subject H039 $(C)$. The OFF maps at each level were summarized over all the subjects. The sections in $A$ and $B$ correspond to sections 2 and 4 in $C$. $b w$, Body weight; $L G N$, lateral geniculate nucleus.

It was suggested in our previous report that the OFF neurons in the auditory cortex are involved in the temporal integration of auditory information, especially for duration selectivity (He et al., 1997). There are long-duration-selective neurons and durationtuned neurons in the cat auditory cortex as well as in the cat visual cortex (Duysens et al., 1996; He et al., 1997). Although it was not specifically stated in the reports by Casseday and colleagues (Casseday et al., 1994; Ehrlich et al., 1997), some of the durationtuned neurons in the bat inferior colliculus looked like OFF neurons.

For auditory duration integration, the $\mathrm{ON}$ response has been considered as a starting pistol signal. A stop signal is needed to punctuate the integration and is likely provided by the OFF neurons. All OFF neurons in the auditory thalamus are long- duration-selective, although their minimal responsive (Fig. 1D), half-maximal responsive, and saturation durations are different from neuron to neuron (our unpublished results). Showing relative linear slopes in the duration-tuning curves, the OFF neurons might encode the duration information in their spike numbers and provide the base for duration-selective responses of both long-latency and OFF neurons in the auditory cortex (He et al., 1997).

Summarizing the above findings, OFF neurons are associated with the motion of a sound source, are duration-selective, and are thus proposed to relate to the temporal integration of duration information. In addition to the above roles, the OFF neuron is very likely to code the decrement of the intensity of an acoustic signal, as suggested in the visual system for a light signal. 
The physiological implications of the segregation of $\mathrm{ON}$ and OFF layers in the auditory thalamus are unknown. Because the thalamus is the last relay station to the auditory cortex, this separation may help to sort different information in the cortex. Another possible functional role of the segregation is to enable independent processing of the $\mathrm{ON}$ and $\mathrm{OFF}$ information before it reaches the cortex. An exploration of the corticofugal modulatory effects on the $\mathrm{ON}$ and $\mathrm{OFF}$ pathways may provide us with a hint as to whether the processing of different pathways is different and/or independent.

\section{REFERENCES}

Aitkin LM, Prain SM (1974) Medial geniculate body: unit responses in the awake cat. J Neurophysiol 37:512-521.

Bisti S, Gargini C, Chalupa LM (1998) Blockade of glutamate-mediated activity in the developing retina perturbs the functional segregation of ON and OFF pathways. J Neurosci 18:5019-5025.

Bowling DB, Michael CR (1984) Terminal patterns of single, physiologically characterized optic tract fibers in the cat's lateral geniculate nucleus. J Neurosci 4:198-216.

Calford MB, Webster WR (1981) Auditory representation within principal division of cat medial geniculate body: an electrophysiological study. J Neurophysiol 45:1013-1028.

Casseday JH, Ehrlich D, Covey E (1994) Neural tuning for sound duration: role of inhibitory mechanism in the inferior colliculus. Science 264:847-950.

Duysens J, Schaafsma SJ, Orban GA (1996) Cortical Off response tuning for stimulus duration. Vision Res 36:3243-3251.

Ehrlich D, Casseday JH, Covey E (1997) Neural tuning to sound duration in the inferior colliculus of the big brown bat, Eptesicuc fuscus. J Neurophysiol 77:2360-2372.

Grinnell AD (1973) Rebound excitation (off-responses) following nonneural suppression in the cochlear of echolocating bats. J Comp Physiol [A] 82:179-194.

He J (1997) Modulatory effects of regional cortical activation on the onset responses of the cat medial geniculate neurons. J Neurophysiol 77:896-908.

He J, Hashikawa T (1998) Connections of the dorsal zone of cat auditory cortex. J Comp Neurol 400:334-348.

He J, Hashikawa T, Ojima H, Kinouchi Y (1997) Temporal integration and duration tuning in the dorsal zone of cat auditory cortex. J Neurosci 17:2615-2625.

Heil P, Langner G, Scheich H (1992) Processing of frequencymodulated stimuli in the chick auditory cortex analogue: evidence for topographic representations and possible mechanisms of rate and directional sensitivity. J Comp Physiol [A] 171:583-600.

Henry KR (1985) ON and OFF components of the auditory brainstem responses have different frequency- and intensity-specific properties. Hear Res 18:245-251.

Hubel DH, Wiesel TN (1961) Integrative action in the cat's lateral geniculate body. J Physiol (Lond) 155:385-398.

Jassik-Gerschenfeld D, Teulon J, Ropert N (1976) Visual receptive field types in the nucleus dorsolateraris anterior of the pigeon's thalamus. Brain Res 108:295-306.

Jones EG (1985) The thalamus. New York: Plenum.

Kiang NY-S (1965) Discharge patterns of single nerve fibers in the cat's auditory nerve. Research monograph 35. Cambridge, MA: MIT Press.

Le Vay S, McConnell S (1982) ON and OFF layers in the lateral geniculate nucleus of the mink. Nature 300:350-351.

Neuweiler G, Schuller G, Schnitzer H-U (1971) On- and off-responses in the inferior colliculus of the greater horseshoe bat to pure tones. $\mathrm{Z} \mathrm{Vgl}$ Physiol 74:57-63.

Norton TT, Rager G, Kretz R (1985) ON and OFF regions in layer IV of striate cortex. Brain Res 488:348-352.

Pelleg-Toiba R, Wollberg Z (1989) Tuning properties of auditory cortex cells in the awake squirrel monkey. Exp Brain Res 74:353-364.

Rapisarda C, Bacchelli B (1977) The brain of the guinea pig in stereotaxic coordinates. Arch Sci Biol 61:1-37.

Redies H, Brandner S (1991) Functional organization of the auditory thalamus in the guinea pig. Exp Brain Res 86:384-392.

Redies H, Brandner S, Creutzfeldt OD (1989) Anatomy of the auditory thalamocortical system of the guinea pig. J Comp Neurol 282:489-511.

Schiller PT (1982) Central connections of the retinal ON and OFF pathways. Nature 297:580-583.

Schiller PT, Malpeli JG (1978) Functional specificity of lateral geniculate nucleus laminae of the rhesus monkey. J Neurophysiol 41:788-797.

Semple MN, Kitzes LM (1993) Binaural processing of sound pressure level in cat primary auditory cortex: evidence for a representation based on absolute levels rather than interaural level differences. J Neurophysiol 69:449-461.

Singer W, Creutzfeldt OD (1970) Reciprocal lateral inhibition of onand off-center neurons in the lateral geniculate body of the cat. Exp Brain Res 10:311-330.

Spitzer MW, Semple MN (1995) Neurons sensitive to interaural phase disparity in gerbil superior olive: diverse monaural and temporal response properties. J Neurophysiol 73:1668-1690.

Stryker MP, Zahs KR (1983) ON and OFF sublaminae in the lateral geniculate nucleus of the ferret. J Neurosci 3:1943-1951.

Suga N (1964) Single unit activity in cochlear nucleus and inferior colliculus of echo-locating bats. J Physiol (Lond) 172:449-474.

Suga N (1973) Feature extraction in the auditory system of bats. In: Basic mechanisms in hearing (Moller AR, ed), pp 675-744. New York: Academic.

Suga N, Manabe T (1982) Neural basis of amplitude-spectrum representation in auditory cortex of the mustached bat. J Neurophysiol 47:225-255.

Suga N, Simmons JA, Shimozawa T (1974) Neurophysiological studies on echolocation system in awake bats producing CF-FM orientation sounds. J Exp Biol 61:379-399.

Suga N, Simmons JA, Jen PHS (1975) Peripheral specialization for fine analysis of Doppler-shifted echoes in the auditory system of the "CFFM" bat Pteronotus parnellii. J Exp Biol 61:161-192.

Toronchuk JM, Stumft E, Cynader MS (1992) Auditory cortex neurons sensitive to correlates of auditory motion: underlying mechanisms. Exp Brain Res 88:169-180.

Zurita P, Villa AEP, de Ribaupierre Y, de Ribaupierre F, Rouiller EM (1994) Changes of single unit activity in the cat's auditory thalamus and cortex associated to different anesthetic conditions. Neurosci Res 19:303-316. 